\title{
Enhancing the Throughput of FT Mass Spectrometry Imaging Using Compressed Sensing and Subspace Modeling
}

\author{
Yuxuan Richard Xie ${ }^{[1][4]}$, Daniel C. Castro ${ }^{[3][4]}$, Stanislav S. Rubakhin ${ }^{[2][4]}$, Jonathan V. Sweedler ${ }^{[1][2][3[4] *}$, \\ Fan $\operatorname{Lam}^{[1][4] *}$ \\ [1] Department of Bioengineering, University of Illinois at Urbana-Champaign \\ [2] Department of Chemistry, University of Illinois at Urbana-Champaign \\ [3] Department of Molecular and Integrative Physiology, University of Illinois at Urbana-Champaign \\ [4] Beckman Institute for Advanced Science and Technology, University of Illinois at Urbana-Champaign
}

\begin{abstract}
Mass spectrometry imaging (MSI) allows for untargeted mapping of the chemical compositions of tissues with attomole detection limits. MSI using Fourier transformbased mass spectrometers, such as FT-ion cyclotron resonance (FT-ICR), grants the ability to examine the chemical space with unmatched mass resolution and mass accuracy. However, direct imaging of large tissue samples on FT-ICR is restrictively slow. In this work, we present an approach that combines the subspace modeling of ICR temporal signals with compressed sensing to accelerate high-resolution FT-ICR MSI. A joint subspace and sparsity constrained reconstruction enables the creation of highresolution imaging data from the sparsely sampled and short-time acquired transients. Simulation studies and experimental implementation of the proposed acquisition in investigation of brain tissues demonstrate a factor of 10 enhancement in throughput of FT-ICR MSI, without the need for instrumental or hardware modifications.
\end{abstract}

Mass spectrometry imaging (MSI) allows for label-free spatially-resolved molecular mapping of biological tissues with exceptional sensitivity and specificity. ${ }^{1-3}$ Particularly, Fourier transform-mass spectrometry (FTMS) offers superb mass resolution desirable for resolving and identifying the molecular contents in chemically complex samples. ${ }^{4,5}$ Atmospheric pressure (AP) matrix-assisted laser desorption/ionization (MALDI) MSI coupled with an Orbitrap was reported to achieve $1.4 \mu \mathrm{m}$ lateral resolution and $\sim 2$ ppm mass accuracy, demonstrating the possibility of high-mass accuracy imaging at the subcellular level. ${ }^{6}$ A $21 \mathrm{~T}$ FT-ICR mass spectrometer was used in an MSI experiment and resolved lipid signals with a molecular mass difference of $1.79 \mathrm{mDa}^{7}$ Despite these significant achievements, a major bottleneck for MSI on FTMS (either on Orbitrap or FT-ICR) is the limited throughput due to the long transient acquisition time needed to resolve isobaric ions and hundreds of thousands of acquisitions required for largescale tissue imaging. The time involved limits the number of acquisitions for an FTMS experiment, especially for high lateral resolution imaging (e.g. for subcellular level details), volumetric imaging, and high-throughput molecular biopsy imaging in clinical settings. Although faster speed can be achieved by several recent hardware developments, ${ }^{8-11}$ imaging acceleration often requires highly customized instrumentation that is not easily accessible.

Computational imaging has played transformative roles in improving the throughput of various imaging modalities. With the unique data complexity and data dimensionality brought by the high-resolution FT-ICR instrument, there is tremendous potential for leveraging computational imaging strategies to address the limitation in throughput for high-mass resolution MSI experiments. For example, subspace imaging has recently been adapted to accelerate FT-ICR MSI by enabling high-resolution reconstruction from short-time acquired transients. ${ }^{12}$ However, as the transient acquisition time per pixel decreases, the measurement overhead (e.g., stage movement, laser firing and ion accumulation) becomes more dominant and limiting the achievable acceleration factor if scanning through all the pixels is still required. Furthermore, the pixelby-pixel subspace fitting strategy does not incorporate prior spatial information and can be sensitive to noise and inaccuracy of the subspace estimation.

Here, we demonstrate an enhanced approach that integrates compressed sensing (CS) with subspace imaging to further increase the FTMS acquisition speed. CS has proven successful in a variety of imaging applications allowing image reconstruction from sparsely sampled data, 13-17 whereas its applicability for FTMS MSI has not yet been explored. The CS allows for spatial sparse sampling complementary to the short temporal transient sampling offered by subspace imaging. The synergy of the two methods allows us to significantly reduce the total FTMS MSI acquisition time. The proposed imaging approach is illustrated in Figure 1A. We accelerate the acquisition by (a) randomly scanning only a fraction of spatial locations and (b) acquiring short transients at the sampled locations. A direct visualization of the reduced data generated by the accelerated acquisition showed ion images lacking informative spatial structures and molecular distributions due to missing data at non-sampled pixel locations and poor spectral resolution, whereas the data reconstructed by our proposed approach displayed significantly improved image features and mass accuracy (Figure 1B). To recover the full high-mass resolution data from this reduced dataset, we developed an integrated subspace and sparsity model-based strategy that jointly reconstructs high-resolution mass 
spectra for all the pixels. Specifically, we modeled the underlying high-dimensional MSI data using a subspace representation $\boldsymbol{\rho}=\boldsymbol{C} \boldsymbol{\Phi}$, in which $\boldsymbol{\Phi}$ is a low-rank matrix containing basis transients whose linear combination accurately approximates the desired long transient (with rank $L \ll$ dimensions of $\boldsymbol{\rho}$ ) and $\boldsymbol{C}$ is also a rank $L$ matrix containing pixel-dependent spatial coefficients (see the Supporting Information on computational details). The reduced data $\boldsymbol{D}$ can be modeled by an underdetermined linear system $\boldsymbol{D}=\boldsymbol{\Omega} \boldsymbol{C} \boldsymbol{\Phi}$ (Figure 1A) with the measurement matrix $\boldsymbol{\Omega}$ describing the spatio-temporal sampling process to generate the reduced data (see Supporting Information for formulation details). The subspace model transforms the problem of directly recovering the high-dimensional $\boldsymbol{\rho}$ into recovering the coefficients matrix $\boldsymbol{C}$, which has a much lower degrees-of-freedom. ${ }^{18,19}$ However, a direct leastsquares reconstruction is ill-posed due to the spatial sparse sampling.

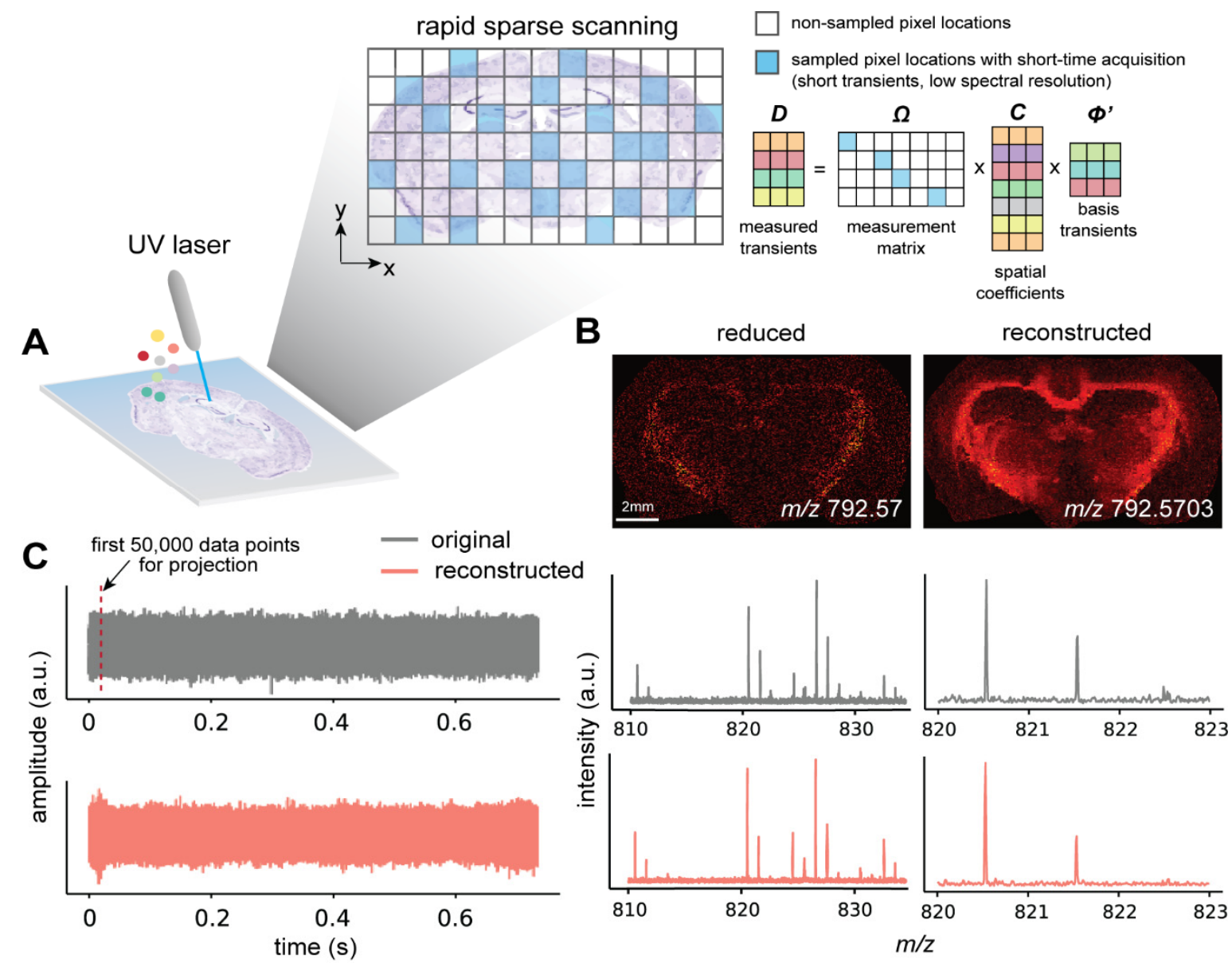

Figure 1. Overview of the proposed imaging approach for enhanced throughput FT-ICR MSI. (A) An illustration of the rapid scanning strategy integrating both the compressed sensing based spatial sparse sampling and the subspace model based short-time acquisition. The data matrix, $D$, contains collected transients with much fewer temporal points per transient than standard acquisition, at randomly sampled pixel locations (corresponding to different color shadings) defined by the measurement matrix (blue grids are 1 and white grids are 0 ). With the predetermined basis transient in $\Phi$, the reconstruction of entire high-resolution imaging dataset is defined as estimating the much lowerdimensional spatial coefficients given the sparsely measured data in $D$. (B) An ion image (left) directly formed from a sparsely sampled dataset (40\% pixels) provides limited interpretation about the tissue morphological features and the spatial distributions of molecules (unsampled pixels filled with zeros). The reconstructed ion image (right) effectively recovers tissue features with a much higher mass accuracy for the corresponding ion signal. (C) An original transient (top left) and a reconstructed transient (bottom left) by projecting the first 50,000 data points of the original transient onto the subspace spanned by a set of basis transients exhibit highly similar spectral profiles and consistent intensity levels (right).

We further exploited the spatial sparsity constraint complementary to the subspace model, formulating the subspace coefficient estimation as the following $\ell 1$ regularized reconstruction problem:

$$
\min _{\boldsymbol{C}}\left\|\boldsymbol{D}-\boldsymbol{\Omega} \boldsymbol{C} \boldsymbol{\Phi}^{\prime}\right\|_{F}^{2}+\lambda\left\|\boldsymbol{W} \boldsymbol{M}^{T} \boldsymbol{C}\right\|_{1} .
$$

The first term enforces data consistency, and the second term encourages a sparse solution in the transform domain. We used wavelet transform operator $\boldsymbol{W}$ as the sparsifying transform to demonstrate the concept while other types of transforms better suited for MSI data could also be used. $\boldsymbol{M}^{T}$ is the image formation operator to map individual pixels to their two-dimensional spatial coordinates (accounting for how the MSI scan is performed), and $\boldsymbol{\Phi}^{\prime}$ is a temporally truncated basis transient matrix from $\boldsymbol{\Phi}$, which is estimated through singular value decomposition (SVD) on a small set of long transients (details in the Supporting Information). We showed that projecting a small number of temporal data points onto the basis transients can faithfully reconstruct the 
original high-resolution mass spectra (Figure 1C), thus enabling significantly shorter data acquisition per transient to achieve the target mass resolution. We solved the optimization problem in eq.(1) using the alternating direction method of multipliers (ADMM). ${ }^{20}$ The algorithmic implementations can be found in the Supporting methods. It should be noted that the described approach without spatial sparse sampling will be a spatially regularized jointsubspace fitting of all pixels simultaneously, which offers improved robustness to noise and subspace estimation error compared to the traditional pixel-by-pixel fitting.
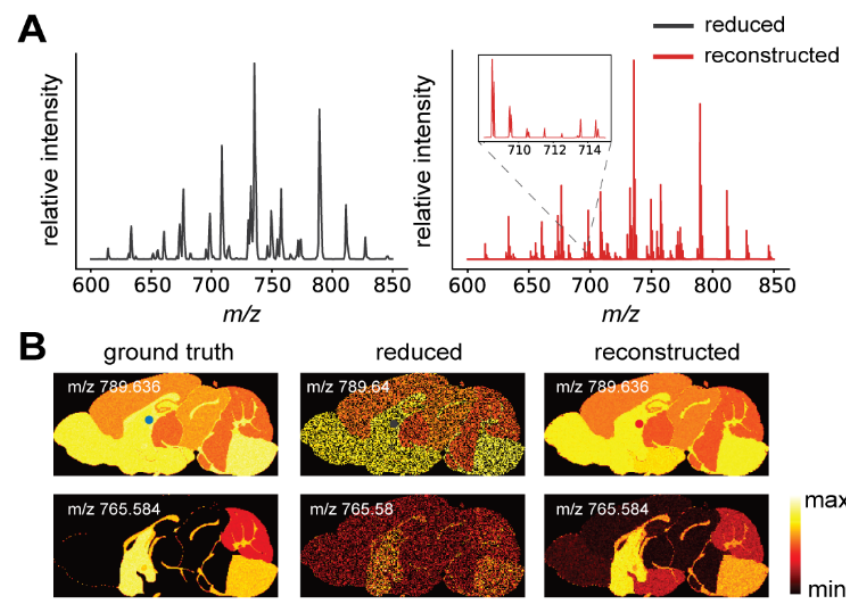

reduced
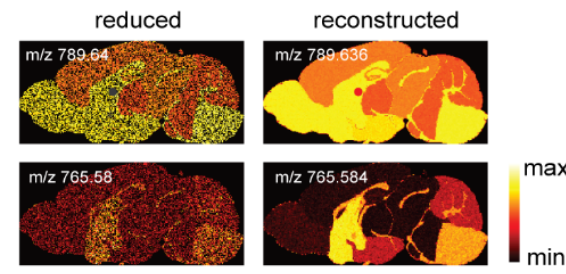

C
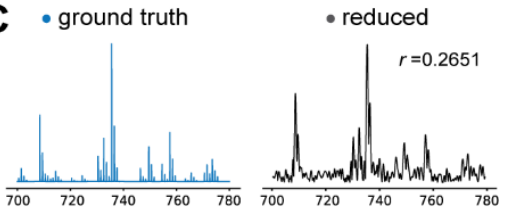

- reconstructed

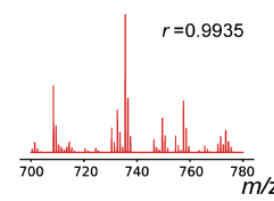

Figure 2. Evaluation of the approach using a simulated FT-ICR MSI dataset. (A) Average spectra of the reduced data (left, $60 \%$ pixels sampled) and the reconstructed data (right) from the same reduced data. Spectral features with close $\mathrm{m} / \mathrm{z}$ values can be resolved after reconstruction (inset). (B) Representative ion images from the ground truth, from the reduced noisy data, and from the reconstructed data for two simulated ions. (C) Individual spectra of the ground truth, the reduced, and the reconstructed data from a selected pixel location. Pearson correlation coefficients are used to evaluate the fidelity to the ground truth spectra.

We first evaluated the method on a simulated FT-ICR MSI dataset, allowing direct comparison of reconstruction against a ground truth for quantitative and unbiased evaluation of the proposed approach. Briefly, we simulated each transient by following the general signal model introduced by Marshal as the sum of exponentially damped sinusoidal waves at various frequencies that represent ions of different mass to charge ratios..$^{21}$ In total, 30 sum formulas (with adducts and isotopic distributions) were simulated and the relative concentrations that define signal intensities were drawn from probability distributions. We further added spatial complexity by assigning the permuted spectral profiles to different annotated regions of the Allen Brain
Atlas (ABA) reference ${ }^{22}$ to generate realistic looking ion images. The simulation pipeline (Figure S1) and the list of chemical formulas (Table S1) can be found in the Supporting Information. To assess the effect of noise on the basis transients and the reconstruction quality, we simulated two datasets: one without noise as the ground truth, and another with Gaussian white noise added (Figure S2). Two sets of basis transients were extracted from both datasets, denoted as the clean basis and noisy basis (Figure S3). Different numbers of transients were sampled from $30,60,80$ and $100 \%$ total number of pixels, all with truncated transient duration (first 5\% temporal points) as available measurements to mimic the sparse sampling and short-time acquisition (Figure 2A, left). The reconstruction was conducted on the reduced noisy data (Figure 2A, right). Representative ion images from the ground truth data, the reduced data, and the reconstructed data using the noisy basis transients with a $60 \%$ sampling rate were compared (Figure 2B). The reconstruction resulted in high spatial and spectral fidelity to the ground truth, in contrast to the poor image and spectral quality produced directly from the reduced data (Figure 2B, C). To quantitatively evaluate the reconstruction, we computed Pearson correlation coefficients between all pairs of noiseless reference ion images and the corresponding reconstructed images at various sampling rates (Figure S4). Even with a $30 \%$ sampling rate, data can be accurately recovered, indicated by the strong spatial correlation with the ground truth. In an actual MSI experiment from a tissue, a reasonable sampling rate can be applied to enable sparse sampling coupled with the short-time acquisition, which will provide multiplicative acceleration in the imaging speed by exploiting transform sparsity through compressed sensing. Comparing the reconstructed ion images, we noticed a slight overfitting when the noisy basis was used for reconstruction (Figure S4). This highlights the importance of accurate basis estimation for practical experimental data, which can be improved by multiple scans then averaging per pixel for higher signal-to-noise ratios (SNRs).

Next, we demonstrated the capability of our approach through retrospective sparse sampling of fully sampled highresolution MALDI MSI datasets of rat brain sections collected by a 7T FT-ICR mass spectrometer. The basis transients were estimated on a random subset of highresolution transients sampled from a subset of pixel locations as previously described. ${ }^{12} \mathrm{We}$ followed the same reconstruction evaluation procedure used on the simulated dataset. Because the ground truth of the experimental data was unknown, we adapted peak picking on the average mass spectrum to form ion images from the full data as the reference for comparison. Reference and reconstructed ion images of $m / z 820.5359\left(\left[\mathrm{C}_{44} \mathrm{H}_{79} \mathrm{NO}_{10}+\mathrm{K}\right]^{+}, \mathrm{ppm}=2.81\right)$ and $m / z 832.6497\left(\left[\mathrm{C}_{45} \mathrm{H}_{89} \mathrm{NO}_{11}+\mathrm{H}\right]^{+}, \mathrm{ppm}=1.32\right)$ with different sampling rates were shown in Figure 3A. Reconstructing using a $30 \%$ sampling rate displays morphological consistency to the reference ion image (Fig. 3A, second row), 

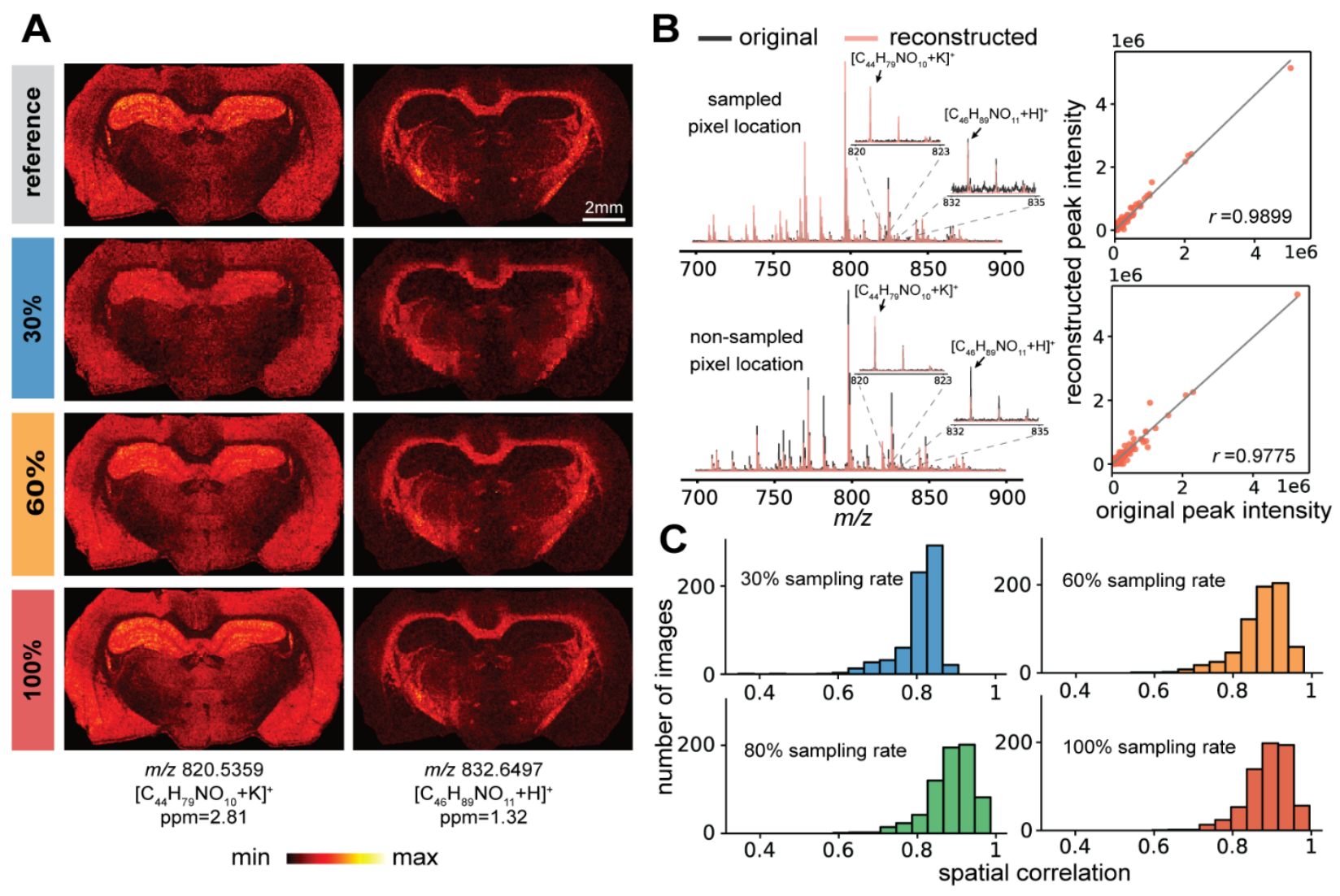

Figure 3. Reconstructing a high-resolution FT-ICR MSI dataset of a rat brain coronal section from reduced data obtained through retrospective sparse sampling. (A) Selected ion images are shown at $m / z 820.5359$ and $\mathrm{m} / \mathrm{z} 832.6497$ from the reference (original data, row 1) and the reconstructed datasets with 30,60 and 100\% pixels sampled (rows 2 to 4 respectively). (B) Mass spectra from the reference and reconstructed data exhibit strong consistency for both sampled and non-sampled pixel locations recovered by the proposed approach. Highlevel similarity between reference and reconstructed mass spectra is supported by quantitative analysis of peak intensity profiles using the Pearson correlation. The insets show the isotopic distributions for two ion signals. (C) The histograms of the spatial correlation measures of ion images from reference and reconstructed data suggest that the proposed method can produce accurate molecular distributions from sparsely sampled data.

though missing some subtle structural details. Using a $60 \%$ sampling rate (Fig. 3A, third row) significantly improves the recovery of finer details. It is worth noting that fully sampling all pixel locations (100\% sampling rate) followed by the proposed reconstruction produces better quality images than the reference due to the denoising effect of the $\ell 1$ regularization (Fig. 3A, fourth row). We are not reconstructing one ion image at a time, but rather exploiting the spatio-spectral redundancy to reconstruct the whole intensity profiles of the mass spectra (Figure 3B). Spectral consistencies were evaluated through the Pearson correlation between the original and reconstructed spectral intensities, which showed strong correlation for both sampled and non-sampled pixel locations (Figure 3B, Figure S5). The overall reconstruction quality determined by the spatial correlation suggests that sampling just close to half of the total number of pixels may be sufficient to achieve high-quality reconstruction (Figure 3C, Figure S5).

To further demonstrate the feasibility of the proposed approach, we experimentally implemented the integrated subspace and CS based sparse sampling strategy on a 7T FTICR mass spectrometer. To better assess the reconstruction quality under practical experimental scenarios, we acquired multiple datasets from the rat brain adjacent slices using different sampling settings with a $25 \mu \mathrm{m}$ raster width (Table 1). A high-resolution dataset (dataset 1 ) was fully sampled with standard transient acquisition

Table 1. Acquisition settings for 6 different experimental datasets used for evaluation.

\begin{tabular}{|c|c|c|c|c|}
\hline Dataset & $\begin{array}{l}\text { Total } \\
\text { number } \\
\text { of } \\
\text { pixels }\end{array}$ & $\begin{array}{c}\text { Number of } \\
\text { pixels sampled }\end{array}$ & $\begin{array}{l}\text { Transient } \\
\text { duration } \\
\text { (s) }\end{array}$ & $\begin{array}{c}\text { Data } \\
\text { acquisition/to } \\
\text { tal imaging } \\
\text { time (min) }\end{array}$ \\
\hline 1 & 99585 & $99585(100 \%)$ & 0.731 & $1213 / 2039$ \\
\hline 2 & 88699 & $88669(100 \%)$ & 0.045 & $66 / 724$ \\
\hline 3 & 100856 & $60513(60 \%)$ & 0.045 & $45 / 497$ \\
\hline 4 & 84918 & $42459(50 \%)$ & 0.045 & $32 / 350$ \\
\hline 5 & 84763 & $33905(40 \%)$ & 0.045 & $25 / 282$ \\
\hline 6 & 86759 & $26027(30 \%)$ & 0.045 & $19 / 219$ \\
\hline \multicolumn{5}{|c|}{$\begin{array}{l}(0.731 \mathrm{~s} \text { acquisition time per transient, } 160,000 \text { mass } \\
\text { resolution at } \mathrm{m} / \mathrm{z} 400) \text {, which took } 34 \mathrm{~h} \text { to complete and was } \\
\text { processed using the standard method. Five other datasets } \\
\text { (datasets } 2-6) \text { were all acquired with a short transient time } \\
(0.045 \mathrm{~s}) \text { and reconstructed using the proposed method. All }\end{array}$} \\
\hline
\end{tabular}


pixels were sampled for dataset 2 . Although sampling only short transients significantly reduced the data acquisition time, measurement overhead became dominant and limited the reduction of the total imaging time. Datasets 3 to 6 were randomly sampled for a portion of the pixels at different ratios (under-sampled data), further reducing the total imaging time on top of the acceleration already offered by the short-time acquisition. Specifically, with a $40 \%$ sampling rate, we were able to acquire and reconstruct a dataset containing 84,763 pixels in $<5$ h (dataset 5). A $30 \%$ sampling rate with short-time acquisition led to an approximately $10 \times$ faster scan than the traditional uniform sampling. Method details on the sample preparation, experimental implementation, data acquisition, and reconstruction are provided in the Supporting Information.

We then qualitatively assessed the reconstruction quality of each individual dataset. Ion images of lipids distributions are shown in Figure 4A. Similar to results shown in Figure 3, images reconstructed by the proposed approach with fully sampled pixel locations but short transients (Figure 4A ii, dataset 2) display even higher SNRs and better contrast in comparison to the images from standard reconstruction of fully sampled datasets acquired using long transients (Figure 4A i). Undersampled data still yielded high-quality reconstruction (Figure 4A iii, iv, Figure S6). Although gradual loss of fine spatial features was
A

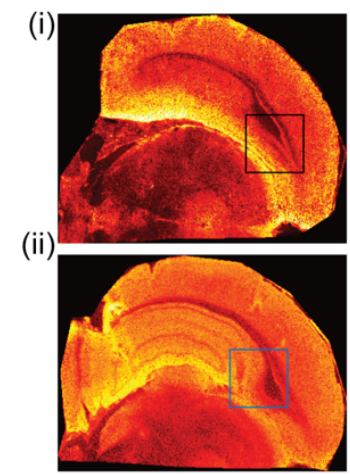

(iii)

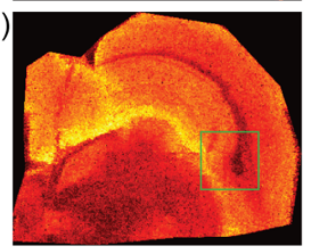

(iv)

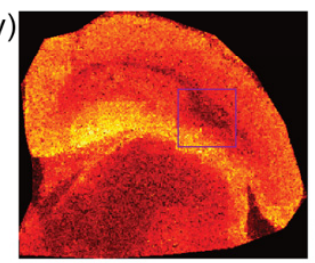

$m / z 756.5513$

$\left[\mathrm{C}_{42} \mathrm{H}_{78} \mathrm{NO}_{8} \mathrm{P}+\mathrm{H}\right]^{+}$ ppm $=3.31$

C

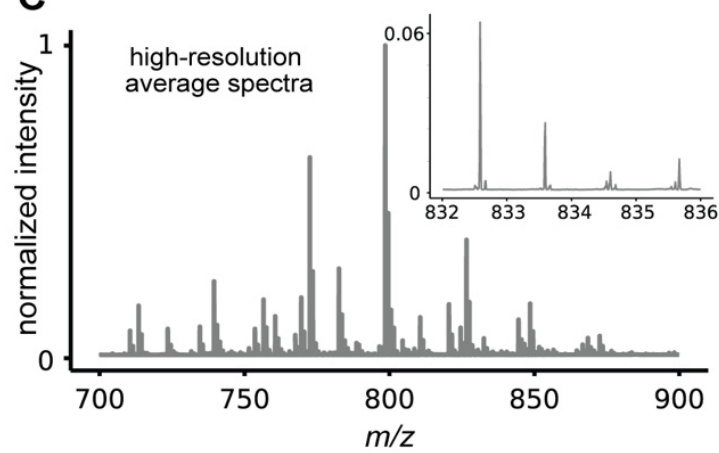

B
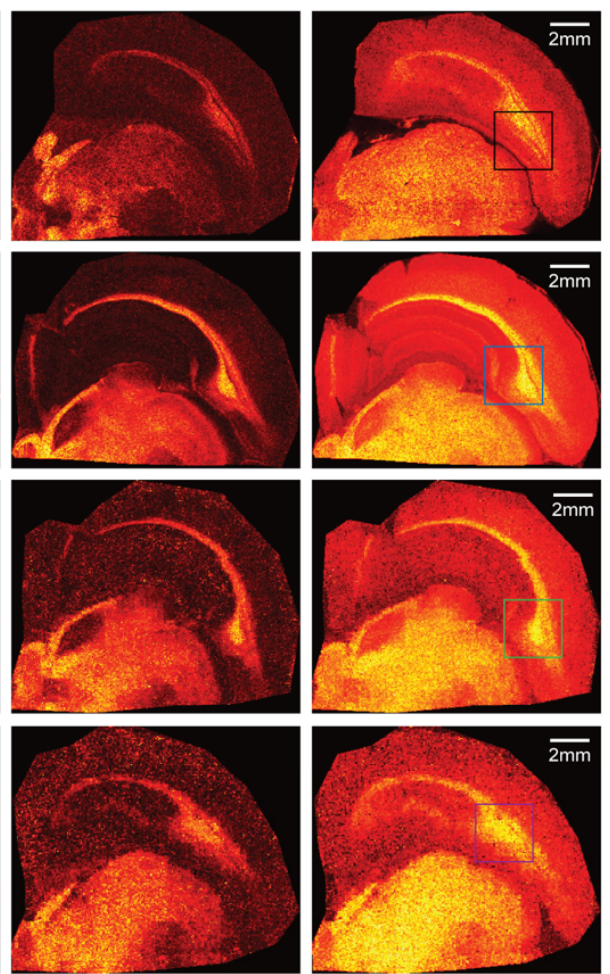

$m / z 810.5789$

$\left[\mathrm{C}_{44} \mathrm{H}_{86} \mathrm{NO}_{7} \mathrm{P}+\mathrm{K}\right]^{+}$

ppm $=1.97$

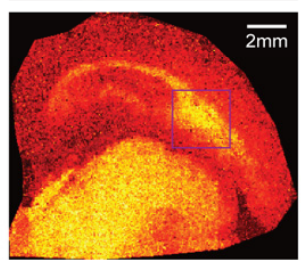

$\mathrm{m} / \mathrm{z} 826.5744$

$\left[\mathrm{C}_{49} \mathrm{H}_{80} \mathrm{NO}_{7} \mathrm{P}+\mathrm{H}\right]^{+}$

ppm $=0.12$
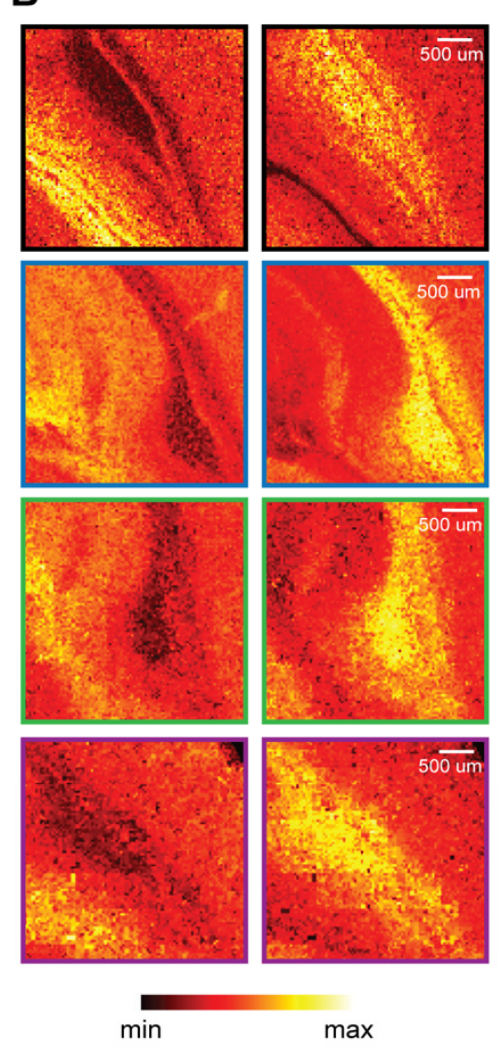

$\min$

$\max$

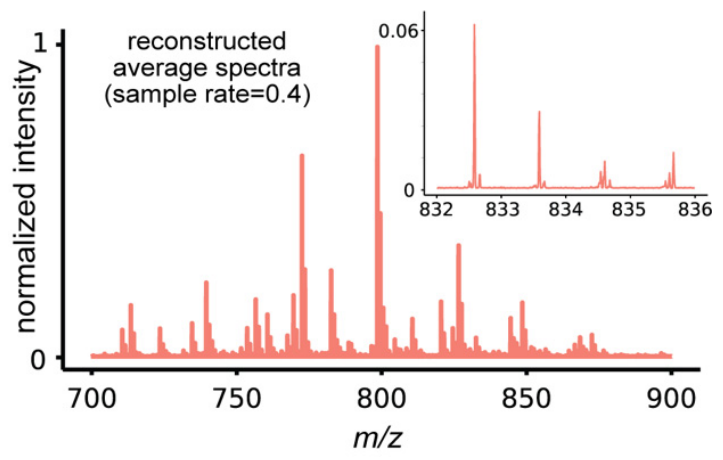

Figure 4. Results from different datasets listed in Table 1, generated by experimental implementation of the proposed sparse sampling strategy and reconstruction through our algorithm. (A) Three representative ion images at $\mathrm{m} / \mathrm{z} 756.5513,810.5789$, and 826.5744 are shown for the (i) fully sampled high-resolution dataset (HR), (ii) short-time acquisition without spatial sparse sampling, (iii) short-time acquisition with $60 \%$ pixels sampled, and (iv) short-time acquisition with $40 \%$ pixels sampled. (B) Zoomed in ion images at $\mathrm{m} / \mathrm{z} 756.5513$ and 826.5744 of brain regions, which include part of the corpus collosum, display substantial recovery of the spatial information and structural details even 
with a relatively low sampling rate. (C) The average mass spectra for the range of $\mathrm{m} / \mathrm{z} 700-900$ from the fully sampled dataset and the reconstructed dataset with $40 \%$ pixels sampled. Insets show monoisotopic distribution for a selected ion in a small $\mathrm{m} / z$ window.

observed as sampling rate decreases, we still found satisfactory recovery of details, e.g., as demonstrated by the zoomed in images of two ions $\left[\mathrm{C}_{42} \mathrm{H}_{78} \mathrm{NO}_{8} \mathrm{P}+\mathrm{H}\right]^{+}$and $\left[\mathrm{C}_{49} \mathrm{H}_{80} \mathrm{NO}_{7} \mathrm{P}+\mathrm{H}\right]^{+}$at the corpus callosum region (Figure $4 \mathrm{~B}$ ) observed in adjacent slices. Additionally, we performed principal component analysis (PCA) on all 6 datasets to unveil the spatial variations of the reconstructed chemical profiles (Figure S7). A considerably low sampling rate $(30 \%)$ could adequately recover spatio-chemical information to allow interpretation of the chemical variations across the tissue.

In summary, we document an integrative computational and experimental approach that integrates CS and subspace imaging based sparse sampling. Although the traditional subspace approach reduces the acquisition time per pixel, the novel combination with CS allows for multiplicative acceleration factors by also reducing the measurement overhead. Results from both simulations and real experimental data demonstrate significantly improved throughput enabled by the proposed approach. Our computational imaging strategy provides more flexibility in balancing the imaging speed, resolution, and data quality. The increased speed either enables more samples to be imaged per instrument or the same number of samples at a higher spatial resolution. Theoretically, for tissues with less structural and chemical complexity than the brain, a lower sampling rate and shorter transient duration than what we practiced in the experiments can be achieved due to a higher redundancy introduced by the sample homogeneity, thus providing an even higher imaging throughput. Our method is applicable to another FT MS analyzer, the Orbitrap, given its similar nature of signals, and can also be integrated with other instrumentation and scanning strategies that minimize measurement overhead for greater acceleration of highresolution, high-throughput FTMS MSI.

\section{Author Information}

\section{Corresponding Author}

Jonathan V. Sweedler - Department of Bioengineering, Department of Molecular and Integrative Physiology, Department of Chemistry, and Beckman Institute for Advanced Science and Technology, University of Illinois at Urbana Champaign, Urbana, Illinois 61801, United States; orcid.org/0000-0003-3107-9922; Email: jsweedle@ illinois.edu

Fan Lam - Department of Bioengineering, Beckman Institute for Advanced Science and Technology, Cancer Center at Illinois, University of Illinois at UrbanaChampaign, Urbana, Illinois 61801, United States; orcid.org/0000-0002-4124-0663; Email: fanlam1@ illinois.edu

\section{Authors}

Yuxuan Richard Xie - Department of Bioengineering and Beckman Institute for Advanced Science and Technology, University of Illinois at Urbana-Champaign, Urbana, Illinois 61801, United States; orcid.org/0000-0003-1664-9114
Daniel C. Castro - Department of Molecular and Integrative Physiology and Beckman Institute for Advanced Science and Technology, University of Illinois at UrbanaChampaign, Urbana, Illinois 61801, United States; orcid.org/0000- 0002-9127-6242

Stanislav S. Rubakhin - Department of Chemistry, Neuroscience Program, and Beckman Institute for Advanced Science and Technology, University of Illinois at Urbana-Champaign, Urbana, Illinois 61801, United States; orcid.org/0000-0003-0437-1493

\section{Data and Code Availability}

The data and code for this paper can be available upon reasonable requests to the authors.

\section{Conflict of Interest}

The authors declare no competing financial interest.

\section{Acknowledgment}

This project was supported by the National Institute on Drug Abuse under Award No. P30DA018310 and the National Human Genome Research Institute under award No. RM1HG010023. Y.R.X acknowledges support from the Beckman Graduate Fellowship. The content is solely the responsibility of the authors and does not necessarily represent the official views of the awarding agencies.

\section{Keywords}

compressed sensing, fast acquisition, imaging, mass spectrometry

\section{REFERENCES}

(1) Norris, J. L.; Caprioli, R. M. Analysis of Tissue Specimens by Matrix-Assisted Laser Desorption/Ionization Imaging Mass Spectrometry in Biological and Clinical Research. Chem. Rev. 2013, 113 (4), 2309-2342. https://doi.org/10.1021/cr3004295. Rae Buchberger, A.; DeLaney, K.; Johnson, J.; Li, L. Mass Spectrometry Imaging: A Review of Emerging Advancements and Future Insights. Anal Chem 2018, 90 (1), 240-265. https://doi.org/10.1021/acs.analchem.7b04733.

(3) Wang, T.; Cheng, X.; Xu, H.; Meng, Y.; Yin, Z.; Li, X.; Hang, W. Perspective on Advances in Laser-Based High-Resolution Mass Spectrometry Imaging. Anal. Chem. 2020, 92 (1), 543-553. https://doi.org/10.1021/acs.analchem.9b04067.

(4) Scigelova, M.; Hornshaw, M.; Giannakopulos, A.; Makarov, A. Fourier Transform Mass Spectrometry. Mol Cell Proteomics 2011, 10 (7). https://doi.org/10.1074/mcp.M111.009431.

(5) Xian, F.; Hendrickson, C. L.; Marshall, A. G. High Resolution Mass Spectrometry. Anal. Chem. 2012, 84 (2), 708-719. https://doi.org/10.1021/ac203191t.

(6) Kompauer, M.; Heiles, S.; Spengler, B. Atmospheric Pressure MALDI Mass Spectrometry Imaging of Tissues and Cells at 1.4Mm Lateral Resolution. Nature Methods 2017, 14 (1), 90-96. https://doi.org/10.1038/nmeth.4071.

(7) Bowman, A. P.; Blakney, G. T.; Hendrickson, C. L.; Ellis, S. R.; Heeren, R. M. A.; Smith, D. F. Ultra-High Mass Resolving Power, Mass Accuracy, and Dynamic Range MALDI Mass Spectrometry Imaging by 21-T FT-ICR MS. Anal. Chem. 2020, 92 (4), 3133-3142. https://doi.org/10.1021/acs.analchem.9b04768.

(8) Prentice, B. M.; Chumbley, C. W.; Caprioli, R. M. High-Speed MALDI MS/MS Imaging Mass Spectrometry Using Continuous 
Raster Sampling. Journal of Mass Spectrometry 2015, 50 (4), 703-710. https://doi.org/10.1002/jms.3579.

(9) Bednařík, A.; Kuba, P.; Moskovets, E.; Tomalová, I.; Krásenský, P.; Houška, P.; Preisler, J. Rapid Matrix-Assisted Laser Desorption/Ionization Time-of-Flight Mass Spectrometry Imaging with Scanning Desorption Laser Beam. Anal. Chem. 2014, 86 (2), 982-986. https://doi.org/10.1021/ac402823n.

(10) Kooijman, P. C.; Nagornov, K. O.; Kozhinov, A. N.; Kilgour, D. P. A.; Tsybin, Y. O.; Heeren, R. M. A.; Ellis, S. R. Increased Throughput and Ultra-High Mass Resolution in DESI FT-ICR MS Imaging through New-Generation External Data Acquisition System and Advanced Data Processing Approaches. Scientific Reports 2019, 9 (1), 1-11. https://doi.org/10.1038/s41598-01836957-1.

(11) Spraggins, J. M.; Caprioli, R. M. High-Speed MALDI-TOF Imaging Mass Spectrometry: Rapid Ion Image Acquisition and Considerations for Next Generation Instrumentation. $J \mathrm{Am} \mathrm{Soc}$ Mass Spectrom 2011, 22 (6), 1022-1031. https://doi.org/10.1007/s13361-011-0121-0.

(12) Xie, Y. R.; Castro, D. C.; Lam, F.; Sweedler, J. V. Accelerating Fourier Transform-Ion Cyclotron Resonance Mass Spectrometry Imaging Using a Subspace Approach. J. Am. Soc. Mass Spectrom. 2020, 31 (11), 2338-2347.

https://doi.org/10.1021/jasms.0c00276.

(13) Lustig, M.; Donoho, D.; Pauly, J. M. Sparse MRI: The Application of Compressed Sensing for Rapid MR Imaging. Magnetic Resonance in Medicine 2007, 58 (6), 1182-1195. https://doi.org/10.1002/mrm.21391.

(14) Zhu, L.; Zhang, W.; Elnatan, D.; Huang, B. Faster STORM Using Compressed Sensing. Nature Methods 2012, 9 (7), 721-723. https://doi.org/10.1038/nmeth.1978.

(15) Ma, Y.; Lee, Y.; Best-Popescu, C.; Gao, L. High-Speed Compressed-Sensing Fluorescence Lifetime Imaging Microscopy of Live Cells. PNAS 2021, 118 (3). https://doi.org/10.1073/pnas.2004176118.

(16) Cleary, B.; Simonton, B.; Bezney, J.; Murray, E.; Alam, S.; Sinha, A.; Habibi, E.; Marshall, J.; Lander, E. S.; Chen, F.; Regev, A. Compressed Sensing for Highly Efficient Imaging Transcriptomics. Nature Biotechnology 2021, 1-7. https://doi.org/10.1038/s41587-021-00883-x.

(17) Bartels, A.; Dülk, P.; Trede, D.; Alexandrov, T.; Maaß, P. Compressed Sensing in Imaging Mass Spectrometry. Inverse Problems 2013, 29 (12), 125015. https://doi.org/10.1088/0266$5611 / 29 / 12 / 125015$.

(18) Liang, Z. SPATIOTEMPORAL IMAGINGWITH PARTIALLY SEPARABLE FUNCTIONS. In 2007 4th IEEE International Symposium on Biomedical Imaging: From Nano to Macro; 2007; pp 988-991. https://doi.org/10.1109/ISBI.2007.357020.

(19) Lam, F.; Ma, C.; Clifford, B.; Johnson, C. L.; Liang, Z.-P. HighResolution 1H-MRSI of the Brain Using SPICE: Data Acquisition and Image Reconstruction. Magnetic Resonance in Medicine 2016, 76 (4), 1059-1070. https://doi.org/10.1002/mrm.26019.

(18) Yang, J.; Zhang, Y. Alternating Direction Algorithms for 1-1 Problems in Compressive Sensing. SIAM J. Sci. Comput. 2011, 33 (1), 250-278. https://doi.org/10.1137/090777761.

(21) Marshall, A. G.; Hendrickson, C. L.; Jackson, G. S. Fourier Transform Ion Cyclotron Resonance Mass Spectrometry: A Primer. Mass Spectrometry Reviews 1998, 17 (1), 1-35. https://doi.org/10.1002/(SICI)1098-2787(1998)17:1<1::AIDMAS1>3.0.CO;2-K

(22) Sunkin, S. M.; Ng, L.; Lau, C.; Dolbeare, T.; Gilbert, T. L.; Thompson, C. L.; Hawrylycz, M.; Dang, C. Allen Brain Atlas: An Integrated Spatio-Temporal Portal for Exploring the Central Nervous System. Nucleic Acids Research 2013, 41 (D1), D996D1008. https://doi.org/10.1093/nar/gks1042. 


\section{Table of Contents}

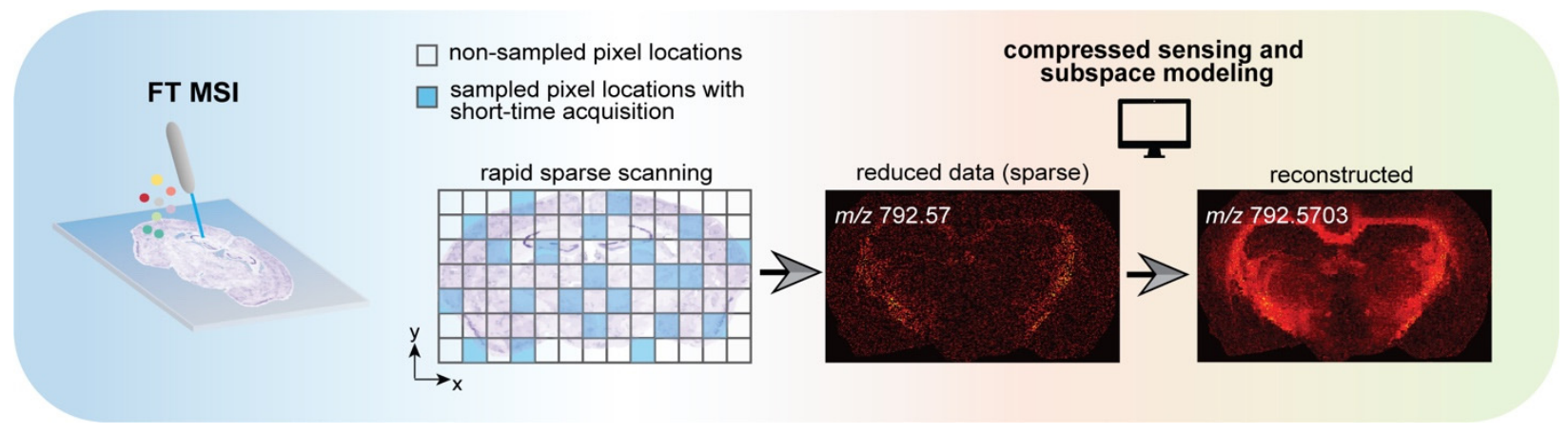

Faster mass spectrometry imaging: A computational imaging method that integrates compressed sensing and subspace modeling enables high-throughput mass spectrometry imaging using Fourier transform- mass spectrometry of large tissue sections. The method allows faithful reconstruction of high-dimensional MSI data while providing up to an order of magnitude faster imaging speed.

Institute twitter: @BeckmanInst, @BIOENGatIL, @ChemistryUIUC

Research twitter: @RichardYuxuan 